\title{
Green Networks and Green Tariffs as Driven by User Service Demand
}

\author{
Louis-Francois Pau \\ Copenhagen Business School \& Rotterdam School of Management \\ lpau@nypost.dk
}

\begin{abstract}
This section describes an approximate model built from real subsystem performance data, of a public wireless network (3G / LTE) in view of minimum net energy consumption or minimum emissions per time unit and per user. This approach is justified in order to generate the integrated view required for "green" optimizations, while taking into account service demand and operations While subsystems with lower native energy footprints are being migrated into public networks, the many adaptation mechanisms at sub-system, protocol and management levels make system complexity too high to design major comprehensive "green" trade-offs. However, by focusing on the incremental effects of a new network user, the approximate model allows marginal effects to be estimated with good accuracy. This capability allows for the provisioning of personalized energy / emissions reducing tariffs to end users with inherent advantages both to operators, energy suppliers and users. One key advantage is the possibility to reduce waste capacity, and thus energy consumption in the network, by allowing the user to specify just the service capacity and demands he/she has. From an engineering point of view, the incremental model allows to tune sub-system characteristics jointly, especially transceivers, transmission and storage. From a configuration point of view, the model allows to determine which nodes in the network benefit most from back-up and renewable power sources. From a business perspective, the model allows to determine trade-offs between personalized bundle characteristics and the energy cost share in the marginal operating expense share. Detailed sub-system model and design improvements are carried out on a continuous basis in collaboration with industry.
\end{abstract}

Keywords: Wireless networks, Energy consumption, CO2 emissions, Marginal analysis, Personalized tariffs, Green wireless tariffs.

\section{Introduction and Scope}

Ever since wireless networks have been developed with mobile terminals, the energy consumption of autonomous access terminals as well as of the access infrastructure has been in focus at the design stage, just to ensure the longest possible communications link durations. When later such wireless networks have been introduced by licensed civilian public operators, the energy expenses of the access and backbone infrastructures have turned into a growing component of their operating expenses, 
creating the need for thorough energy consumption analysis. This analysis has especially focused on the spatially decentralized layout (and thus power supply) of base stations and transmission links, and on coding/modulation schemes. Later on, operators and equipment suppliers alike have put more and more emphasis on the $\mathrm{CO} 2$ emissions from network and device operations, as well as on recycling and sustainable designs ; this last move has so far been motivated by a combination of corporate citizenship, competitive "green" branding, and pressure for better device and system designs. In this evolution, very little attention has been given to the changing nature of the communications traffic carried by the public wireless networks. Likewise the shift in part away from voice calls and messaging, to mobile data with growing effective bandwidth, has not yet impacted wireless network energy analysis. And, most importantly, the user has not been incentivized to drive their traffic and services demands in a way leading to energy and emissions reductions, ultimately making them responsible in the overall processes. This section addresses therefore the following issues:

1. Characterize the energy consumption and emissions jointly of public wireless network infrastructure and of the actual user-selected service derived traffic;

2. Characterize wireless infrastructure technology migration effects on energy and emissions (from UMTS to HSPDA and LTE);

3. Offer a tool to industry allowing to design basic and value-added service tariffs in view of eventual investments in renewable energy production, and of the introduction of "green" wireless telecommunications tariffs.

It has been chosen in this section not to include mobile terminals' and smart phone's own energy consumption and emissions patterns, as the variability thereof is very big due to: technology (incl. operating systems), designs, usage, and battery properties over time. That contribution from terminals to emissions is known to be an order of magnitude less than the one from the networks. The unique service needs enabled by smart phones is addressed through value-added services. Also, in view of the high complexity in network architecture, technologies and operations, approximations have been made sufficient to address issues 1-3 above, which differs from contributions to specific devices, coding schemes, or protocols.

Part 2 addresses the modeling methodology, part 3 the approximate wireless operator energy model, part 4 some examples of numerical results, and part 5 their use for green wireless tariffs. As this work was done for and with industry, there are no public references.

\section{$2 \quad$ Modeling Methodology}

It is obviously very difficult with present state-of-the art to build detailed engineering models calculating precisely the overall energy consumption or emissions of a complete wireless network in operations. This difficulty is not unique to the field, in that it is shared with many other large scale systems serving users with mostly services: e.g., transportation, logistics, national economies, and power grids themselves. 
However, microeconomic theory comes to the rescue, in that it proposes not to build macro-models for prediction, but marginal models for an individual user assuming an approximate model of the global system, and that the individual user is representative of the aggregate set of users. In other words, the marginal models aim at modeling as accurately as possible, for a given context, the effects of one typical user on the first or second order derivatives of the global model.

This approach can be expanded to consider a distribution of individual users, even with outliers, to estimate the distributions of the marginal effects of a given user, while preserving the fundamental properties of the approximate global model.

In the context of wireless green networks, the following marginal indicators driven by user generated traffic and service requests are especially important:

- the individual user's marginal contribution to total network energy consumption (over a given time frame);

- the individual user's marginal contribution to total $\mathrm{CO} 2$ emissions (over a given time frame); and

- the individual user's marginal contribution to network energy costs, network operating costs (OPEX), and network capital expenditure (CAPEX) in that and traffic request.

The above cost elements can be offset against the incremental revenues dictated by the tariff plan allowing for the requested service and traffic provisioning, so that the network operator's incremental profit (or loss) can be determined. In this section, rather than letting the operator fix the tariff plans, it is the user who specifies his request (generic services, value-added service, contract duration, and maximum price), and let's operators bid for a contract with him/her, according to a reverse auction process. In this way, excess capacity in the wireless network and the corresponding energy consumption can be reduced, in that the subscriber does normally not want to pay for service capacity or offerings he/she do not intend to use. Therefore, the analysis and modeling rests on the following main tasks:

- Build with industry data an approximate network infrastructure and traffic model, with energy footprints for all main subsystems (radio, transmission , cooling) and traffic dependent energy consumption (circuit switched and IP); the network allows the provisioning of a set of services (generic and value-added) to a subscriber base;

- Structure the individual user's contributions to CAPEX, OPEX, Billing, CRM, Network management, Content acquisition and net energy costs, as a model of the marginal flows linked to one additional user, on top of an existing subscriber base;

- Include a reverse auction bidding process, whereby the incremental user states the service duration, his/hers basic bundle price offer, and his/hers value-added bundle offer for user-set service duration; operator then must select and configure network resources accordingly allowing fine-tuning of tariffs and incentives with profitability and emissions constraints. 


\section{Approximate Wireless Operator Energy Model}

This part describes an approximate model built from real sub-system performance data, of a public wireless network (3G / LTE) in view of minimum net energy consumption or minimum emissions per time unit and per user. The computational implementations exist at different levels: static non-linear, non-linear with average traffic intensities, randomized over users by Monte-Carlo simulation, and determination of energy /emissions levels by value at risk.

\subsection{Services and Infrastructure}

A detailed and complex realistic approximate model of a wireless service supplier has been designed and built since 2006 with heavy industry involvement, for the limited purpose described in part 2. This model has served in a variety of projects and has shown its flexibility and reasonable accuracy. The services which can be provisioned include:

- Common services: Network management, billing from CDR and IPDR/CRM;

- Generic services: voice, SMS/MMS and metered mobile IP traffic;

- Sample Value-added service: for illustration is used the M-Singing service where a user download songs, and has interactive comments by a paid employee to improve performance;

- Other value-added services studied: Mobile video, technical wireless field support, public ticketing;

Assuming an aggregated subscriber base number, and specified network coverage, the following input-output sub-models have been built on the basis of subsystem data, performance, and energy/cooling consumption supplied by 8 equipment suppliers or operators:

- UTRAN (Radio): RBS and Node-B's for UMTS, EDGE/HSPDA, LTE (100 Mbit);

- Transmission: line cards, Microwave links, ATM over IP, WDM, SONET;

- Backbone: MGW, edge routers, core routers, AAA, signaling;

- Storage: CDR, billing/CRM data, on-demand media, regulated security records;

- Power: electrical grid power supply, local wind power at base station, local solar power at base station, backup local power; and

- Cooling for most large sub-systems.

Network capacity is balanced to meet minimum QoS thresholds (effective bandwidth, blocking probability, content storage capacity) from the aggregate subscriber base assume to have service and traffic request as the individual user. Excess capacity not used by generic wireless services (voice, messaging, mobile Internet) may be used by value-added services; if it is insufficient, extra capacity is provisioned by SLA's at higher usage costs. 
Another essential property of the approximate operator model is that it is not technology dependent, in that it is the service and traffic mix request of the user which drives the access technology whose parameters are used. In other words, and with a simplistic illustration: if the individual user only wants a voice service, the access network parameters will be those of a $3 \mathrm{G}$ infrastructure supplying him with that service at his required QoS levels, supplemented by a common backbone. Vice-versa, if the individual user only wants a value-added service with high data rates and content access, the access network parameters are likely to be those of an LTE access network. As a result of this property, the reverse auction process drives technology switching in the access network, with associated performances, costs and of course energy consumption.

Obviously, the quality of the approximate model hinges on sub-system data or measurements. Real technical input-output data (power, volume, voltage, frequency, performance) were obtained in most cases, from 8 different worldwide suppliers, for different technologies / generations. When relevant, published statistical regression results (from estimated or usage sub-system data) produced by various research groups or companies were also used. Building premises and eventual separate data center models have been available but not yet incorporated.

\subsection{Energy Consumption, Renewables, and Emissions}

Each sub-system has normally an energy consumption which is the sum of a static design related part, and of a sub-system usage part, with in addition cooling. As an example of the usage part for radio transceivers, the energy consumption of each is determined to be at a power level driven by both the inter-base station ranges, and by the channel related capacity driven by traffic; further parameters such as frequency range, and spectrum efficiency and modulation types are accounted for if data were obtained.

All core infrastructure, real-time storage and backup transmission links operate on in-sourced electrical grid power. In addition, an exogenous parametric proportion of distributed nodes (RBS, TRX, links) with renewable energy sources (wind, photovoltaic) is assumed. These renewables obviously impact favorably $\mathrm{CO} 2$ emissions as already demonstrated by some operators. A minimum electrical grid or backup supply (batteries or generators) for all distributed nodes is provisioned, in relation to infrastructure nodes' and local traffic power consumption (incl. cooling).

The mix wind/ solar is parametric, but the operator is assumed to own and operate such sources with the full imputation of the corresponding investments to CAPEX. Excess renewable power supply from the wireless network is sold at eventually subsidized rates, reducing operator's total OPEX. Here data on performances and costs were obtained from two Danish suppliers of renewable energy systems. 


\section{$4 \quad$ Numerical Results on Individual User Energy Consumption and Emissions Driven by Personalized Services and Tariffs}

The numerical calculations required for the calculation of the specified energy and emissions indicators are quite heavy. The further estimation of the probability distributions of these indicators on the basis of distributions of user requests mandates the use of a high performance or grid computing environment. In this part we will only give two cases corresponding to two personalized user requests in a given wireless operator environment, and some parametric curves of some indicators with regards to the user bidding prices for the generic services and the chosen value-added service (which affect the wireless technology GSM, EDGE, UMTS, HSPDA, LTE used to supply the personalized demand).

The operator environment has the following properties: 10 Million subscribers, tele-density of 576 users/ cell, typical average power consumption / user /month of 18 $\mathrm{kWh}$, share of RBS with renewable sources of $15 \%$.

Case 1: Contract duration of 3 months; user bid for generic services of 50 Euros; user bid for MSinging service of 100 Euros.

With this demand profile, the traffic is mostly carried by EDGE, and the emissions indicators are:

- $\mathrm{CO} 2$ emissions driven by capacity in $\mathrm{kg} / \mathrm{user} /$ contract: 20.02

- $\mathrm{CO} 2$ emissions driven by capacity and generic services in $\mathrm{kg} / \mathrm{user} /$ contract: 21.05

- $\mathrm{CO} 2$ emissions driven by generic services in $\mathrm{kg} / \mathrm{user} /$ contract: 0.58

- $\mathrm{CO} 2$ emissions driven by value-added service in $\mathrm{kg} / \mathrm{user} /$ contract: 0.45

- Renewable energy resold by the network during contract duration: 403581 Euros

Case 2: Contract duration of 1 month; user bid for generic services of 260 Euros; user bid for MSinging service of 50 Euros.

With this demand profile, the traffic is mostly carried out by LTE (100 Mb), and the emissions indicators are:

- $\mathrm{CO} 2$ emissions driven by capacity in $\mathrm{kg} / \mathrm{user} /$ contract: 6,57

- $\mathrm{CO} 2$ emissions driven by capacity and generic services in $\mathrm{kg} / \mathrm{user} /$ contract: 7,09

- $\mathrm{CO} 2$ emissions driven by generic services in $\mathrm{kg} / \mathrm{user} /$ contract: 0,52

- $\mathrm{CO} 2$ emissions driven by value-added service in $\mathrm{kg} / \mathrm{user} /$ contract: 0

- Renewable energy resold by the network during contract duration: 194071 Euros

In general the analysis of results proves that here are very many interactions to account for. Although $\mathrm{CO} 2$ emissions due to infrastructure capacity/coverage dominate, the share of generic services and especially of value-added services grows rapidly with service/content richness and QoS real-time performances. While taking into account spectral system efficiency and frequency bands, individual emissions get slightly smaller with newer technologies (HSPDA, LTE), subject mostly to design and microelectronics progress; critical is the mix of low emission green technologies in RBS nodes. 


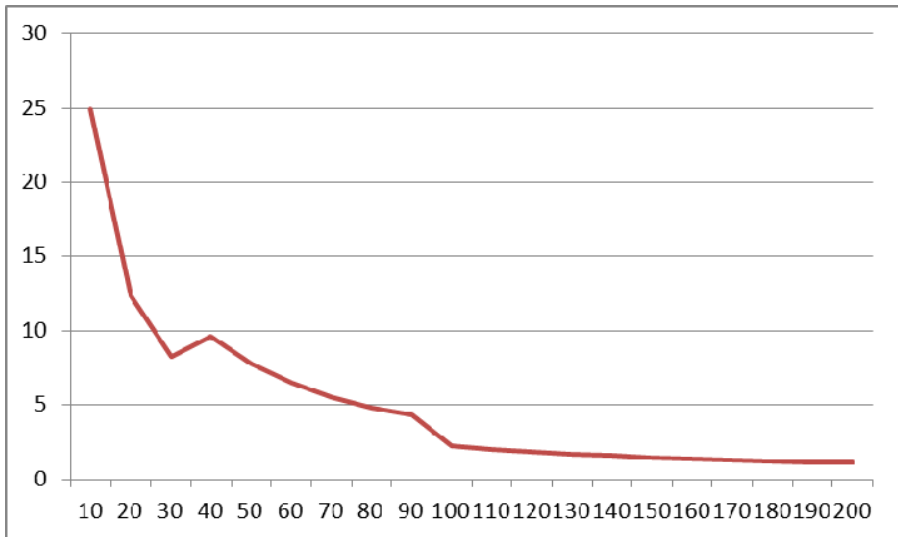

Fig. 1. Net Energy costs per user in \% of total OPEX vs. generic services bundle price in Euros; assumes contract duration of 3 months, and a value added service bid of 100 Euros

\section{$5 \quad$ Green Wireless Tariffs}

We define here a "green wireless tariff", as the contractual price paid by a subscriber to an operator for a set of services at a given quality of service for a specified duration; this price must reflect both parties best efforts to reduce $\mathrm{CO} 2$ emissions, and pass onto the subscriber a share of the energy and emissions savings in the network. The operator can easily offer "green wireless tariffs" by having a green tariff subscriber base, which, against contracting such plans, commits the operator to earmark a specified percentage of their energy consumption at network level to technologies with fewer emissions and possibly to the use of renewable energy supplies.

If furthermore, such green wireless tariffs are made personalized, they provide an incentive to users to specify and adopt a wireless service demand profile leading to less wasted or unutilized capacity, as unutilized capacity burns energy. As this very fact is likely to have a relatively more drastic effect, there is a strong likelihood that personalized green wireless tariffs are to be become very important.

Their deployment however rests on equilibrium between users requesting personalization, and operator's economic sustainability as they then have to make new investments in technology and/or renewable power sources they control. The analysis made possible by the model described in this section shows that operator's profit are not eroded and sufficient to sustain such investments for generic services (Figure 3); however, for value-added services, the situation is less clear as they also demand investments in content, storage, competence (Figure 4). The revenues at network level from the resale to the grid of excess renewable electrical power, obviously increases profits, even if some are shared with subscribers to green wireless tariff plans. 


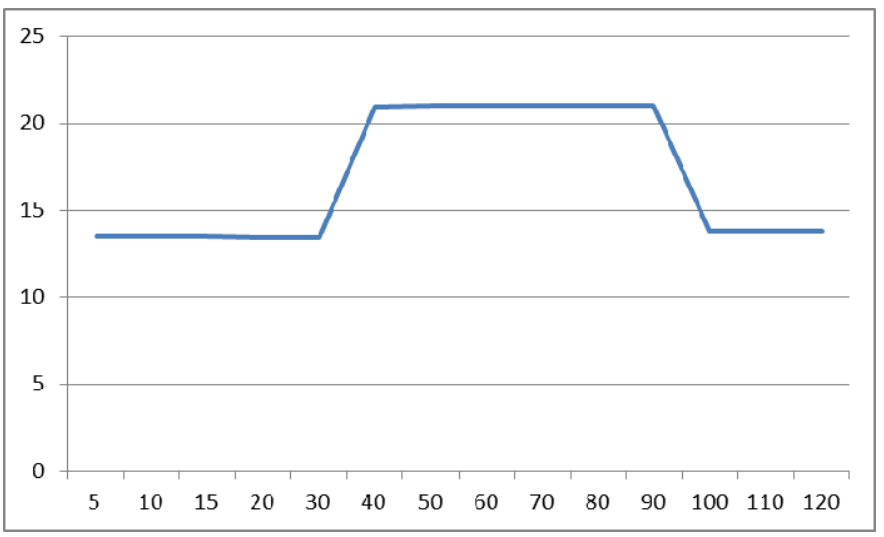

Fig. 2. $\mathrm{CO} 2$ emissions per user in $\mathrm{kg} \mathrm{CO} 2$ vs. generic services bid in Euros (access terminals excluded); this assumes contract duration of 3 months, and a value-added service bid of 100 Euros

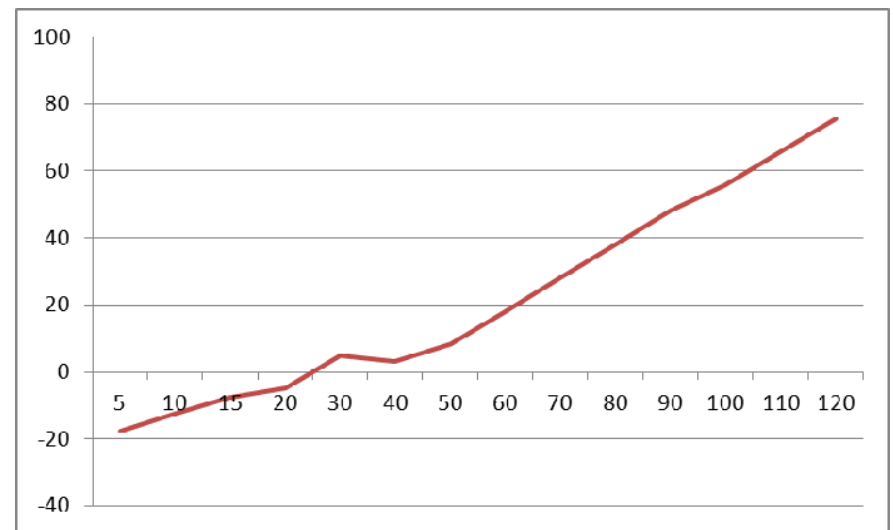

Fig. 3. Net incremental profit per user from personalized green tariff vs. generic service offer in (Euros) for contract duration; contract duration is 3 months and value-added service offer is 100 Euros 


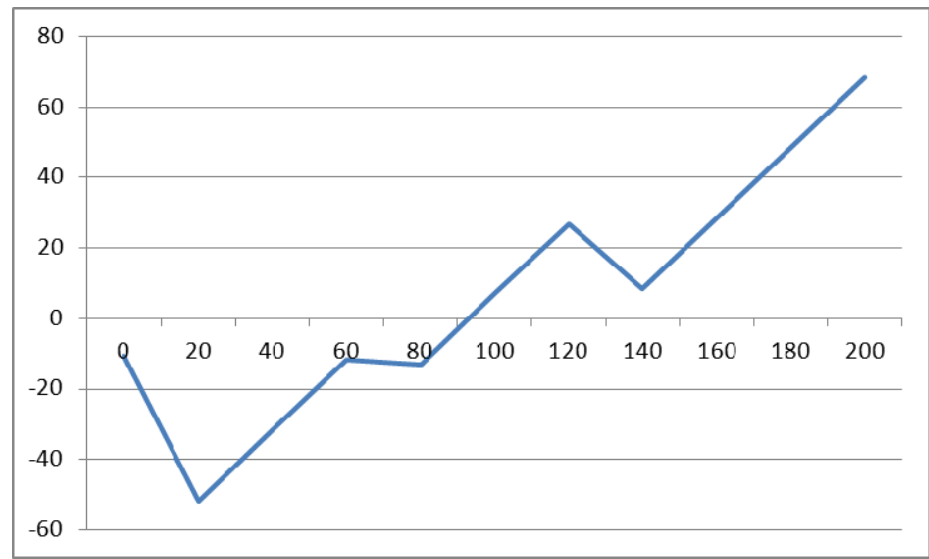

Fig. 4. Net incremental profit per user from personalized green tariff vs. value-added service offer in (Euros) for contract duration; contract duration is 3 months and generic services offer is 50 Euros

In summary, new "green" tariffs can be designed with: (a) personalized service characteristics reducing wasted network capacity, and (b) incentivizing users to larger renewable power supply grades by OPEX bonuses. The impact is though mostly from and for high traffic/ content users.

\section{Conclusions and Future Research ${ }^{1}$}

By modeling and analyzing wireless networks energy consumption and emissions in order to make explicit and quantify individual user's impact, this section has shown that this integrated modeling approach is necessary as each incremental user has a significant impact. It is also necessary to be able to design and implement "green wireless tariffs" and their personalized versions which reduce waste in network utilization.

Open Access. This article is distributed under the terms of the Creative Commons Attribution Noncommercial License which permits any noncommercial use, distribution, and reproduction in any medium, provided the original author(s) and source are credited.

\footnotetext{
${ }^{1}$ Due to the nature of this study no references can be provided.
} 